\title{
The Clinical Features and Outcomes of Patients with Takotsubo Syndrome: The Experience at an Emergency General Hospital
}

\author{
Takuya Nagata and Masahiro Mohri
}

\begin{abstract}
:
Objective We aimed to elucidate clinical characteristics, contemporary practice and outcomes of patients with takotsubo syndrome who were hospitalized in an emergency general hospital with angiography capabilities.

Methods This retrospective study included a total of 42 consecutive patients who were admitted between January 2010 and August 2014.

Results The study population included 11 men (26\%) and 31 women (74\%) [median age 76 years (interquartile range, 66-83)]. Physical stress and emotional stress were identified as triggers in 28 (67\%) patients and $5(12 \%)$ patients, respectively. Electrocardiographic changes were observed in 41 (98\%) patients, with ST-segment elevation being the most common (71\%) finding. In-hospital complications occurred in $24(57 \%)$ patients, and acute pulmonary congestion or cardiogenic shock was seen in $21(50 \%)$ patients. Five patients died during hospitalization (in-hospital mortality: 12\%).

Conclusion Takotsubo syndrome was associated with significant morbidity and mortality among elderly patients who were treated at an emergency general hospital. Physicians and surgeons in all departments should be aware of the condition, especially in acutely ill subjects.
\end{abstract}

Key words: takotsubo syndrome, takotsubo cardiomyopathy, elderly, emergency general hospital

(Intern Med 57: 641-645, 2018)

(DOI: 10.2169/internalmedicine.9249-17)

\section{Introduction}

Takotsubo syndrome is an acute disorder characterized by transient left ventricular dysfunction, which most commonly affects the apex. It is often preceded by an emotional or physical trigger. Postmenopausal women are preferentially affected, and the clinical presentation, electrocardiographic findings, and biomarker profiles often resemble those of acute coronary syndrome (1-3). Recent studies have reported life-threatening complications including left ventricular free wall rupture, left ventricular outflow tract obstruction, thrombosis and cardiogenic shock, and the in-hospital or long-term outcomes are not necessarily as benign as previously reported $(3,4)$. In the present study, we aimed to elucidate the contemporary practice and outcomes of takotsubo syndrome in patients who were hospitalized in our hospital, which is a general hospital with angiography capabilities that is located in Kitakyushu city. Kitakyushu city is an industrial city with a population of approximately 972,000 ; individuals of $\geq 65$ years of age account for $29 \%$ of the total resident population, while those of $\geq 75$ years of age account for $14 \%$. It is considered to be among the most aging metropolises in Japan.

\section{Materials and Methods}

A total of 42 consecutive patients with takotsubo syndrome who were admitted to Japan Community Healthcare Organization Kyushu Hospital between January 2010 and August 2014 were retrospectively studied. The diagnosis of takotsubo syndrome was based - in principle - on the Heart

Department of Cardiology, Japan Community Healthcare Organization Kyushu Hospital, Japan

Received: March 27, 2017; Accepted: June 18, 2017; Advance Publication by J-STAGE: November 20, 2017

Correspondence to Dr. Takuya Nagata, tanagata-kyu@umin.org 
Table 1. Baseline Characteristics.

\begin{tabular}{lr}
\hline Age, years & $76[66,83]$ \\
Female gender & $31(74 \%)$ \\
SBP, mmHg & $112[90,144]$ \\
HR, bpm & $88[76,106]$ \\
Hypertension & $24(57 \%)$ \\
Diabetes & $8(19 \%)$ \\
Dyslipidemia & $11(26 \%)$ \\
Current smoker & $8(19 \%)$ \\
Mental disorder & $3(7 \%)$ \\
Malignant neoplasms & $2(5 \%)$ \\
Elevated troponin and/or CK-MB at the onset & $30(71 \%)$ \\
& \\
Electrocardiogram & \\
ST elevation & $30(71 \%)$ \\
ST depression & $13(31 \%)$ \\
Negative T wave & $12(29 \%)$ \\
long QT (QTc $>0.44$ sec) & $32(76 \%)$ \\
normal & $1(2 \%)$ \\
& \\
LVEF at diagnosis & \\
Atypical wall motion abnormalities & $3.42 \pm 0.16$ \\
Symptom at presentation & $3(7 \%)$ \\
Chest pain & \\
SOB & \\
No complaints & $20(5 \%)$ \\
others & \\
\hline Dre & \\
\hline
\end{tabular}

Data are shown as number (percentage) of patients, median [interquartile range] or mean \pm SD.

HR: heart rate, LVEF: left ventricular ejection fraction, SBP: systolic blood pressure, SOB: shortness of breath

Failure Association of the European Society of Cardiology diagnostic criteria for takotsubo syndrome (1). These include: 1) transient regional wall motion abnormalities of left ventricle frequently proceeded by a stressful trigger (emotional or physical); 2) left ventricular contraction abnormality extending beyond the geographic territory of a single coronary artery; 3) the absence of obstructive atherosclerotic coronary artery stenosis when coronary angiography is performed; 4) new and reversible electrocardiographic abnormalities; 5) elevated serum natriuretic peptide (BNP or NTproBNP); 6) positive (but a relatively small elevation) for cardiac troponin; and 7) recovery of the ventricular systolic function on cardiac imaging at follow-up (3-6 months). Inhospital medical treatment and cardiac catheterization were provided at the discretion of physicians-in-charge. The data are shown as the median [interquartile range (IQR)] for continuous variables, and the number (percentage) for categorical data. The Mann-Whitney U test and Fischer's exact test were used as appropriate. All of the statistical analyses were performed using the GraphPad Prism 7 software program (GraphPad Software, San Diego, USA).

\section{Results}

Table 1 summarizes the clinical characteristics of the studied patients. Eleven (26\%) patients were men and 31 $(74 \%)$ women. The age of the patients ranged from 10 months to 91 years [median age 76 years (IQR, 66-83)]. Coronary angiography was performed in 33 patients (79\%). In the remaining nine patients, the diagnosis was made based on the echocardiographic findings of a left ventricular apical ballooning-like dilation in the acute phase and the late normalization of the left ventricular function. STsegment elevation was the most common electrocardiographic finding and was seen at presentation in $30(71 \%)$ patients. ST-segment depression, negative $\mathrm{T}$ wave and long QT (QTc >0.44 s) occurred in 13 (31\%), 12 (29\%) and 32 (76\%) patients, respectively. Chest pain (48\%) and shortness of breath $(31 \%)$ were the most common symptoms on presentation. Seven patients had no symptoms and the occurrence of takotsubo syndrome was initially suggested by electrocardiography. Table 2 shows the possible triggers in the 42 patients. Physical stress and emotional stress were recognized in 28 patients $(67 \%)$ and in 5 patients $(12 \%)$, respectively. Medical illnesses were common and showed wide variation; they included respiratory, gastrointestinal, gynecological, neurological, endocrinological, ophthalmological and orthopedic disorders (Table 2). Nine patients were initially admitted to departments other than cardiology. The median peak serum creatine kinase concentration during hospitalization was 224 (IQR, 183-368) mg/dL. In-hospital complications occurred in $24(57 \%)$ patients, and acute pulmonary congestion and cardiogenic shock were seen in $9(21 \%)$ and $14(33 \%)$ patients, respectively (Table 3 ).

Catecholamines were administered to $12(29 \%)$ patients, while mechanical circulatory support by intra-aortic balloon pumping or extracorporeal membrane oxygenation was necessary in $3(7 \%)$ patients. Respiratory support using a ventilator with endotracheal intubation and non-invasive positive pressure ventilation were required in $9(21 \%)$ and $2(5 \%)$ patients, respectively.

Five patients died during hospitalization (in-hospital mortality, $12 \%$; cardiogenic shock, $n=3$; left ventricular free-wall rupture, $\mathrm{n}=1$; non-cardiovascular comorbidity, $\mathrm{n}=1$ ). The age, systolic blood pressure, heart rate at the onset of symptoms, the prevalence of cardiac risk factors, the prevalence of physical and emotional triggers, the concentration of peak serum creatine kinase level, left ventricular ejection fraction at the diagnosis and the prevalence of complications did not differ between the survivors and the non-survivors to a statistically significant extent (Table 4). In addition, the abovementioned clinical variables and outcomes did not differ between patients with an emotional or unknown trigger and those with a physical trigger. 
Table 2. Possible Triggers.

\begin{tabular}{|c|c|}
\hline Physical stress & $28(67 \%)$ \\
\hline Respiratory disorders & $9(21 \%)$ \\
\hline Bacterial pneumoniae & $7(17 \%)$ \\
\hline Interstitial pneumonia & $1(2 \%)$ \\
\hline Neuromuscular respiratory failure & $1(2 \%)$ \\
\hline Abdominal/Gynecological disorders & $5(12 \%)$ \\
\hline Cholangitis & $2(5 \%)$ \\
\hline Superior mesenteric artery embolism & $2(5 \%)$ \\
\hline Torsion of ovarian tumor pedicle & $1(2 \%)$ \\
\hline Constipation & $1(2 \%)$ \\
\hline Central nervous system disorders & $2(5 \%)$ \\
\hline Cerebral hemorrhage & $1(2 \%)$ \\
\hline Norovirus encephalitis & $1(2 \%)$ \\
\hline Endocrinological /Metabolic disorders & $2(5 \%)$ \\
\hline Diabetic ketoacidosis & $1(2 \%)$ \\
\hline Renal failure & $1(2 \%)$ \\
\hline Others & $3(7 \%)$ \\
\hline Deep vein thrombosis & $1(2 \%)$ \\
\hline Anaphylactic shock induced by chemotherapy & $1(2 \%)$ \\
\hline Fever of unknown origin & $1(2 \%)$ \\
\hline Surgery/Invasive procedures & $2(5 \%)$ \\
\hline Excision of a left atrial myxoma & $1(2 \%)$ \\
\hline Bronchoscope & $1(2 \%)$ \\
\hline Orthopedical disorders/Trauma & $4(10 \%)$ \\
\hline Bone fractures & $2(5 \%)$ \\
\hline Crush syndrome & $1(2 \%)$ \\
\hline Eyeball rupture & $1(2 \%)$ \\
\hline Emotional stress & $5(12 \%)$ \\
\hline Unknown & $9(21 \%)$ \\
\hline
\end{tabular}

Data are shown as number (percentage) of patients.

\section{Discussion}

In the present study, we summarized the clinical features and short-term outcomes of 42 patients with takotsubo syndrome who were diagnosed at an emergency general hospital located in an aging metropolis of Japan. In comparison to previous reports, our study revealed several unique features. First, our patients were older in comparison to the patients in previous reports. The median age was 76 years old and a quarter of the study population was over 83 years of age. In contrast, the mean or median age of the patients enrolled in the previous studies was $<70$ years $(2,3,5-8)$. Second, the rate of in-hospital mortality among our patients was relatively high. Previous studies described takotsubo syndrome as a benign disorder (in-hospital mortality, $\leq 1 \%)(6,9)$; however a recent study using data from a large
Table 3. Managements and In-hospital Outcomes.

\begin{tabular}{lr}
\hline Complications & $24(57 \%)$ \\
Acute pulmonary congestion & $9(21 \%)$ \\
Cardiogenic shock & $14(33 \%)$ \\
Acute pulmonary congestion or cardiogenic shock & $21(50 \%)$ \\
Atrial fibrillation & $4(10 \%)$ \\
NSVT & $1(2 \%)$ \\
VF & $1(2 \%)$ \\
CAVB & $1(2 \%)$ \\
Sinus arrest & $1(2 \%)$ \\
& \\
Managements & \\
Catecholamine use & $12(29 \%)$ \\
IABP & $2(5 \%)$ \\
PCPS & $1(2 \%)$ \\
Temporary pacemaker & $2(5 \%)$ \\
Ventilation with endotracheal intubation & $9(21 \%)$ \\
NIPPV & $2(5 \%)$ \\
& \\
In hospital death & $5(12 \%)$ \\
Cardiogenic shock & $3(7 \%)$ \\
Cardiac rupture & $1(2 \%)$ \\
Pneumoniae & $1(2 \%)$ \\
\hline Data are shown as number (percentage) of patients. & \\
CAVB: complete atrioventricular block, IABP: intra-aortic balloon pump- \\
ing, NIPPV: non-invasive positive pressure ventilation, NSVT: non-sus- \\
tained ventricular tachycardia, PCPS: percutaneous cardiopulmonary sup- \\
port, VF: ventricular fibrillation
\end{tabular}

patient registry demonstrated that takotsubo syndrome was associated with several life-threatening complications and that the in-hospital mortality rate was $4.1 \%$ (3). Moreover, Citro et al. reported that the outcome of elderly ( $>75$ years) patients presenting takotsubo syndrome was poor, with acute heart failure occurring in $22.9 \%$ of the patients and an inhospital mortality rate of $6.3 \%$ (vs. $1.5 \%$ in patients of $<75$ years of age) (7). In our study, acute pulmonary congestion or cardiogenic shock occurred in $50 \%$ of the patients and the in-hospital mortality rate was $12 \%$. The high mortality rate in the present study was most likely due to the advanced age of the patients and the presence of concomitant morbidities that occur in association with aging. Third, the major triggers that caused takotsubo syndrome in our patients were physical stresses related to a wide variety of medical illnesses. The prevalence of physical and emotional triggers varied considerably among previous reports $(3,6,10)$. The high rate of patients with a physical trigger in the present study may be related to the serious background of the patients admitted to our hospital, which is designated as an emergency medical and critical care center.

\section{Perspectives}

A wide variety of medical and surgical disorders are managed in general hospitals, and our analysis suggests that takotsubo syndrome can occur in any critically ill patient. Chest symptoms are the most common symptom in these 
Table 4. Comparisons of Clinical Characteristics, Complications and Managements between Hospital Survivors and Non-survivors.

\begin{tabular}{|c|c|c|c|}
\hline & Survivors $(\mathrm{n}=37)$ & Non-Survivors $(n=5)$ & $\mathrm{p}$ value \\
\hline Age & $76[66,82]$ & $75[74,84]$ & 0.48 \\
\hline Female gender & $27(73 \%)$ & $4(80 \%)$ & $>0.99$ \\
\hline Hypertension & $22(59 \%)$ & $2(40 \%)$ & 0.64 \\
\hline Diabetes & $6(16 \%)$ & $2(40 \%)$ & 0.24 \\
\hline Dyslipidemia & $11(30 \%)$ & $0(0 \%)$ & 0.30 \\
\hline Smoke & $7(19 \%)$ & $1(20 \%)$ & $>0.99$ \\
\hline Mental disorder & $2(5 \%)$ & $1(20 \%)$ & 0.32 \\
\hline Carcinoma & $2(5 \%)$ & $0(0 \%)$ & $>0.99$ \\
\hline Biomarker positive at the onset & $26(70 \%)$ & $4(80 \%)$ & $>0.99$ \\
\hline ST elevation $(\geq 1 \mathrm{~mm})$ & $25(68 \%)$ & $5(100 \%)$ & 0.30 \\
\hline Negative $T$ wave & $12(32 \%)$ & $0(0 \%)$ & 0.30 \\
\hline long QT (QTc>0.44) & $29(78 \%)$ & $3(60 \%)$ & 0.58 \\
\hline Within normal range & $1(3 \%)$ & $0(0 \%)$ & $>0.99$ \\
\hline LVEF at diagnosis, $\%$ & $41 \pm 15$ & $45 \pm 23$ & 0.49 \\
\hline Atypical LV wall motion & $3(8 \%)$ & $0(0 \%)$ & $>0.99$ \\
\hline \multicolumn{4}{|l|}{ Symptom at presentation } \\
\hline Chest pain & $17(46 \%)$ & $3(60 \%)$ & 0.66 \\
\hline SOB & $11(30 \%)$ & $2(40 \%)$ & 0.64 \\
\hline No complaints & $7(19 \%)$ & $0(0 \%)$ & 0.57 \\
\hline others & $2(5 \%)$ & $0(0 \%)$ & $>0.99$ \\
\hline Complications & $19(51 \%)$ & $5(100 \%)$ & 0.06 \\
\hline Acute pulmonary congestion & $9(24 \%)$ & $0(0 \%)$ & 0.57 \\
\hline Cardiogenic shock & $9(24 \%)$ & $3(60 \%)$ & 0.13 \\
\hline Acute pulmonary congestion or cardiogenic shock & $18(49 \%)$ & $3(60 \%)$ & $>0.99$ \\
\hline Atrial fibrillation & $4(11 \%)$ & $0(0 \%)$ & $>0.99$ \\
\hline NSVT & $1(3 \%)$ & $0(0 \%)$ & $>0.99$ \\
\hline VF & $0(0 \%)$ & $1(20 \%)$ & 0.12 \\
\hline \multicolumn{4}{|l|}{ Managements } \\
\hline Catecholamine use & $9(24 \%)$ & $3(60 \%)$ & 0.13 \\
\hline IABP & $1(3 \%)$ & $1(20 \%)$ & 0.23 \\
\hline PCPS & $0(0 \%)$ & $1(20 \%)$ & 0.12 \\
\hline Temporary pacemaker & $1(3 \%)$ & $1(20 \%)$ & 0.23 \\
\hline Ventilation with endotracheal intubation & $7(19 \%)$ & $2(40 \%)$ & 0.29 \\
\hline NIPPV & $1(3 \%)$ & $1(20 \%)$ & 0.23 \\
\hline
\end{tabular}

Data are shown as number (percentage) of patients, median[interquartile range] or mean $\pm \mathrm{SD}$.

IABP: intra-aortic balloon pumping, LVEF: left ventricular ejection fraction, NIPPV: non-invasive positive pressure ventilation, NSVT: non-sustained ventricular tachycardia, PCPS: percutaneous cardiopulmonary support, SOB: shortness of breath, VF: ventricular fibrillation

patients and new or presumed new electrocardiographic changes can provide an additional diagnostic clue. Healthcare professionals in all departments should not overlook the possibility of this lethal yet treatable condition.

\section{Conclusion}

Takotsubo syndrome was associated with significant morbidity and mortality in a cohort of elderly patients who were admitted to an emergency hospital. Physicians and surgeons in all departments should be aware of the condition, especially in acutely ill subjects.
The authors state that they have no Conflict of Interest (COI).

\section{References}

1. Lyon AR, Bossone E, Schneider B, et al. Current state of knowledge on Takotsubo syndrome: a Position Statement from the Taskforce on Takotsubo Syndrome of the Heart Failure Association of the European Society of Cardiology. Eur J Heart Fail 18: 8-27, 2016.

2. Ghadri JR, Cammann VL, Templin C. The International Takotsubo Registry: rationale, design, objectives, and first results. Heart Fail Clin 12: 597-603, 2016.

3. Templin C, Ghadri JR, Diekmann J, et al. Clinical features and 
outcomes of Takotsubo (Stress) cardiomyopathy. N Engl J Med 373: 929-938, 2015.

4. De Backer O, Debonnaire P, Gevaert S, et al. Prevalence, associated factors and management implications of left ventricular outflow tract obstruction in takotsubo cardiomyopathy: a two-year, two-center experience. BMC Cardiovasc Disord 14: 147, 2014.

5. Cacciotti L, Passaseo I, Marazzi G, et al. Observational study on Takotsubo-like cardiomyopathy: clinical features, diagnosis, prognosis and follow-up. BMJ Open 2: 2012.

6. Sharkey SW, Windenburg DC, Lesser JR, et al. Natural history and expansive clinical profile of stress (tako-tsubo) cardiomyopathy. J Am Coll Cardiol 55: 333-341, 2010.

7. Citro R, Rigo F, Previtali M, et al. Differences in clinical features and in-hospital outcomes of older adults with tako-tsubo cardio- myopathy. J Am Geriatr Soc 60: 93-98, 2012.

8. Aizawa K, Suzuki T. Takotsubo cardiomyopathy: Japanese perspective. Heart Fail Clin 9: 243-247, 2013.

9. Parodi G, Del Pace S, Carrabba N, et al. Incidence, clinical findings, and outcome of women with left ventricular apical ballooning syndrome. Am J Cardiol 99: 182-185, 2007.

10. Eitel I, von Knobelsdorff-Brenkenhoff F, Bernhardt P, et al. Clinical characteristics and cardiovascular magnetic resonance findings in stress (takotsubo) cardiomyopathy. Jama 306: 277-286, 2011.

The Internal Medicine is an Open Access article distributed under the Creative Commons Attribution-NonCommercial-NoDerivatives 4.0 International License. To view the details of this license, please visit (https://creativecommons.org/licenses/ by-nc-nd/4.0/).

(C) 2018 The Japanese Society of Internal Medicine Intern Med 57: 641-645, 2018 